\title{
Beyond the First Recurrence in Scar Phenomena
}

\author{
D. Wisniacki ${ }^{1,2}$, F. Borondo ${ }^{1}$, E. Vergini ${ }^{3}$, and R. M. Benito ${ }^{4}$ \\ ${ }^{1}$ Departamento de Química, C-IX, Universidad Autónoma de Madrid, Cantoblanco-28049 Madrid, Spain. \\ ${ }^{2}$ Departamento de Física "J.J. Giambiagi", FCEN, UBA, Pabellón 1, Ciudad Universitaria, 1428 Buenos Aires, Argentina. \\ ${ }^{3}$ Dep. de Física, Comisión Nacional de Energía Atómica, Avenida del Libertador 8250, 1429 Buenos Aires, Argentina. \\ ${ }^{4}$ Departamento de Física y Mecánica, E.T.S.I. Agrónomos, Universidad Politécnica de Madrid, 28040 Madrid, Spain.
}

(Received August 12, 1999)

The scarring effect of short unstable periodic orbits up to times of the order of the first recurrence is well understood. Much less is known, however, about what happens past this short-time limit. By considering the evolution of a dynamically averaged wave packet, we show that the dynamics for longer times is controlled by only a few related short periodic orbits and their interplay.

PACS numbers: 05.45.+b, 03.65.Ge, 03.65.Sq

The study of the quantum manifestations of classical chaos is at present a topic of very active research interest [1]. Great advance came from random matrix theory (RMT) which provides an understanding of universal statistical properties of quantum spectra [2]. The most striking departure from RMT described so far in the literature is the phenomenon known as "scar" [3]. This term describes an anomalous localization of quantum probability density along unstable periodic orbits (PO) in classically chaotic systems. Heller showed 绍 the importance of the first recurrences of POs in the scarring effect. In a subsequent work Tomsovic and Heller [5] demonstrated that the semiclassical propagation can be carried out with remarkable precission long after classical fine structure had developed on a scale much smaller than $\hbar$, by computing the corresponding correlation function, $C_{\mathrm{scl}}(t)$, as a sum of contributions of the homoclinic excursions of the PO. This procedure is however cumbersome, since in general many orbits are needed to obtain converged results, and this number increases rapidly with time (see Ref. [5] for details). This picture is greatly simplified if alternatively considering the corresponding averaged dynamics for finite periods of time. In this case, as will be shown in this Letter, a structure of a few short POs emerges that govern the quantum dynamics for times past the first recurrence of the original PO.

Understanding scarring can be tackled from two sides. One way is trying to disentangle the complexity involved in the distribution of individual levels in the spectra of classically chaotic systems [6 8]. For example, in Ref. [8] structures localized on short POs of the stadium were obtained, by considering state correlation diagrams and iteratively removing the parametric interactions (avoided crossings) between the involved eigenstates. The other way consists of approaching the problem in a much more straighforward fashion, by studying how the dynamics of POs induce scars in the eigenfunctions of the system.

Heller's work provided a time-dependent view that shows how recurrences in the short time dynamics of a wave packet along the neighborhood of an isolated PO produces the accumulation of quantum probability density characteristic of scars. Very recently Kaplan and Heller [9] have shown how the use of coherent wave packet sums, decaying as the log-time, leads to enhanced scarring. Later, it was described by some of us 10 how (nonstationary) wave functions highly localized over POs can be constructed from finite time Fourier transform of wave packets. This method allows the calculation of the contribution of each eigenstate to scars.

The time-dependent approach presents the additional advantage of being easily connected with spectroscopy experiments through Fourier transform into the energy domain of the autocorrelation function generated by a test initial wave packet [11]. In the short times limit, recurrences originated by isolated unstable POs in the vicinity of the packet translate into low resolution features of the corresponding spectra (or local density of states) consisting of peaks or bands whose widths are determined by their Lyapunov exponents. In the other limit, corresponding to long times, non-linear effects introduce modulations in these envelopes leading essentially to Porter-Thomas distributions of spectral intensities, although it has been shown recently [12] that the scarring effect is still noticeable in the tail of the distribution. Finally, when the Heisenberg time is reached, individual eigenstates are resolved, and we have essentially the infinite resolution version of the spectrum. In this sense, the overall aspect of chaotic spectra is highly conditioned by the least unstable PO (LUPO) contained in the phase space region spanned by the test wave packet.

In this Letter we consider the case of intermediate times which allow the participation of orbits longer than the LUPO. The associated low resolution spectra split into several components showing evidence of the participation of only a few localized structures corresponding to short POs, which also present their signatures in the correlation function. It is the magnitude of the interaction (in the sense of Ref. [8]) among these localized wave functions and that associated to the LUPO that determines which orbits come into play.

In our study we used the desymmetrized stadium bil- 
liard of radius $r=1$ and enclosed area of $1+\pi / 4$. This system constitutes a paradigm in the study of hard chaos. From the classical point of view it has been demonstrated to be ergodic.

To study the scarring effect of the different stadium POs we closely follow the method described in Ref. [10], which consists in following for finite times the dynamics of a wave packet initially located in the vicinity of a particular PO. In our case we use a harmonic oscillator coherent state (throughout the paper $\hbar$ is set equal to one),

$$
\langle x, y \mid \phi\rangle=\left[\frac{2 \alpha}{\pi}\right]^{1 / 4} \mathrm{e}^{-\alpha\left(x-x_{0}\right)^{2}-\alpha\left(y-y_{0}\right)^{2}} \mathrm{e}^{i\left(P_{x}^{0} x+P_{y}^{0} y\right)},
$$

with $\alpha=30.68$. The time evolution of this state can be followed by projection onto the stadium eigenstates, $|n\rangle$ :

$$
|\phi(t)\rangle=e^{-i \hat{H} t}|\phi(0)\rangle=\sum_{n}|n\rangle\langle n \mid \phi(0)\rangle e^{-i E_{n} t},
$$

which are calculated by the scaling method [13]. Recurrences in the corresponding correlation function, $C(t)=$ $\langle\phi(0) \mid \phi(t)\rangle$, determine the structure in the associated finite resolution spectrum,

$$
I_{T}(E)=\frac{1}{2 \pi} \int_{-\infty}^{\infty} d t C(t) W_{T}(t) e^{i E t}
$$

where a window function, $W_{T}(t)$, has been introduced to eliminate features in $C(t)$ taking place after a given time. Choosing a Gaussian form, $e^{-t^{2} / 2 T^{2}}$, for this window the following expression for $I_{T}(E)$ is obtained

$$
I_{T}(E)=\frac{T}{(2 \pi)^{1 / 2}} \sum_{n}|\langle n \mid \phi(0)\rangle|^{2} e^{-T^{2}\left(E-E_{n}\right)^{2} / 2} .
$$

As shown in Ref. 110], it is also possible to compute the wave functions associated to each low resolution band (or envelope), by means of the expression

$$
\begin{aligned}
\left|\Psi^{E_{0}}\right\rangle & =\frac{1}{2 \pi} \int_{-\infty}^{\infty} d t|\phi(t)\rangle W_{T}(t) e^{i E_{0} t} \\
& =\frac{T}{(2 \pi)^{1 / 2}} \sum_{n}|n\rangle\langle n \mid \phi(0)\rangle e^{-T^{2}\left(E_{0}-E_{n}\right)^{2} / 2} .
\end{aligned}
$$

These band wave functions have been shown to be highly localized along the scarring PO, when the time of the first recurrence is used as the smoothing time [10].

We will concentrate our study in one of the most representative short PO of the desymmetrized stadium; namely, that running along the diagonal joining the two extreme points on both axis, which corresponds to a diamond shape in the full version of the stadium. This PO is presented in the inset to Fig. 1 along with some others which are relevant to our study.

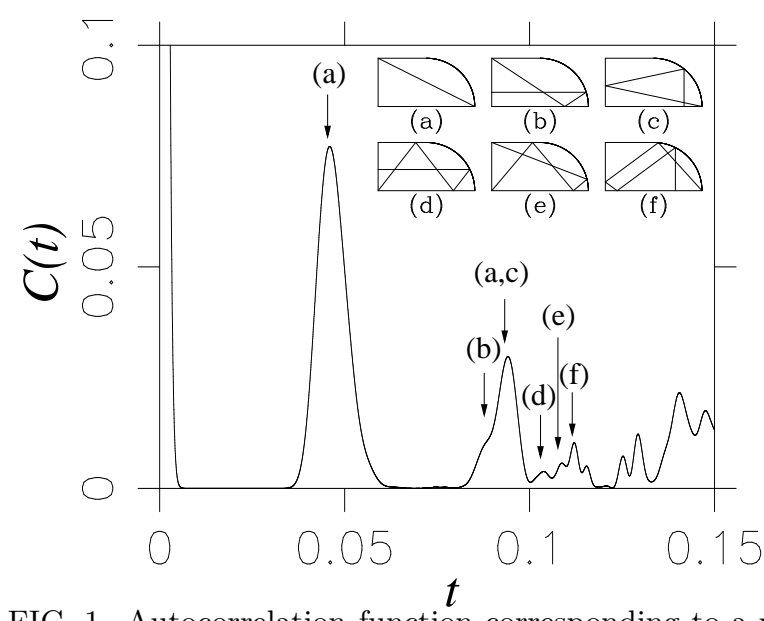

FIG. 1. Autocorrelation function corresponding to a wave packet initially centered on the diagonal periodic orbit (a) of a desymmetrized stadium billiard with $r=1$ and area $1+\pi / 4$. This and other orbits relevant to our study are shown in the inset. Arrows indicate the recurrence times of these periodic orbits.

Using a wave packet [Eq. (11)] initially located on the middle of the diagonal $\mathrm{PO}\left[\left(x_{0}, y_{0}, P_{x}^{0}, P_{y}^{0}\right)=\right.$ $(1,1 / 2,96 / \sqrt{5},-48 / \sqrt{5})]$, the autocorrelation function and the corresponding infinite $(T=\infty)$ and low resolution $(T=0.04)$ spectra have been computed. The results are presented in Figs. 1 and 2, respectively. Notice that the time used in the smoothed version of the spectrum is roughly equal to the period of appearance of the first recurrence in $C(t)$, to which only the diagonal PO contributes. It can be observed that the peaks corresponding to $I_{\infty}$ appear grouped in clumps, so that $I_{T}$ exhibit a series of (seventeen) very well defined bands, which are equally spaced. Moreover, the positions of these peaks, calculated as the mean energy value of each band, are in very good agreement with the energies obtained from the wave numbers, $k$, quantized according to the usual Bohr-Sommerfeld quantization rule:

$$
k=\frac{2 \pi}{L}\left(n+\frac{\nu}{4}\right)
$$

where $n$ is the number of nodes along the orbit, $L=2 \sqrt{5}$ its lenght, and $\nu=9$ the corresponding Maslov index. As an illustration, we show in the upper part of Fig. 2 the wave functions (squared) associated to bands 9 to 12, calculated using Eq. (5). The values of $\sqrt{\langle E\rangle}$ are also given below each plot, so that the agreement with the value predicted by the quantization condition (6) can be checked. As expected, the wave functions appear highly localized along the diagonal PO, corresponding to a number of nodes of 31 to 34 , respectively. This result could have also been obtained with the more elaborated coherent wave packets sums of Kaplan and Heller [9]. 


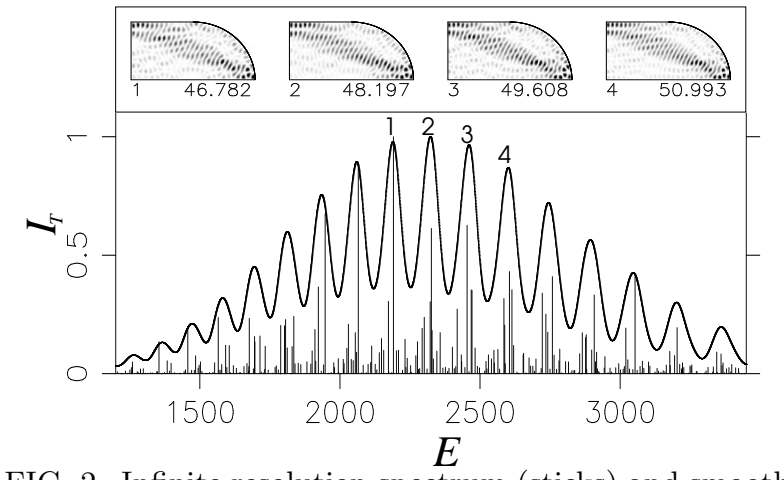

FIG. 2. Infinite resolution spectrum (sticks) and smoothed version of it for $T=0.04$ (full line) corresponding to the autocorrelation function of Fig. 1. In the upper part, the wave functions associated to bands 9 to 12 , calculated using Eq. (月) and $T=0.04$, are presented. The corresponding values of $\sqrt{\langle E\rangle}$ are shown below each plot.

Let us consider now recurrences taking place at longer times in the correlation function of Fig. 1. One can forsee that their analysis will be more complicated; for one thing they appear at longer times so that the packet has had the opportunity to explore more extended regions of phase space (where the linearized dynamics around the $\mathrm{PO}$ is no longer valid), and also several orbits contribute to them. The mechanism responsible for the dynamical coupling of these POs, accessed by the phase space sampling associated to the choice of the initial wave packet (11), is not obvious. Some preliminary results [14] indicate that the dynamics along the manifolds emanating from them and their proximity, is an important factor in this issue.

For this purpose, let us examine the group of peaks in $C(t)$ at $t \sim 0.08-0.12$. It presents a complicated structure; in addition to the main maximum at $t \sim 0.09$, it exhibits a shoulder at smaller time values and four much shorter peaks at longer times. Taking into account the periods of the orbits presented in Fig. 1 a tentative assignment of the contribution to each peak has been made. The result is indicated by labeled arrows over the curve of $C(t)$. To check our hypothesis, we consider a series of low resolution spectra computed using progressively larger smoothing times, so that POs with increasingly longer periods are allowed to come into play. Some representative results, corresponding to the 9-th band of the spectrum of Fig. 2, are presented in Fig. 3. Our calculations show that in the range $T=0.02-0.07$ the smoothed band consists of only one peak, which begins to develop an incipient intraband structure as $T$ increases (see different curves in Fig. 3). After that time the structure gets more clearly defined, so that for the interval $T=0.12-0.20$ four intraband components are readily observable (dotted line in Fig. 3). The associated wave functions are shown in the upper part of the figure.

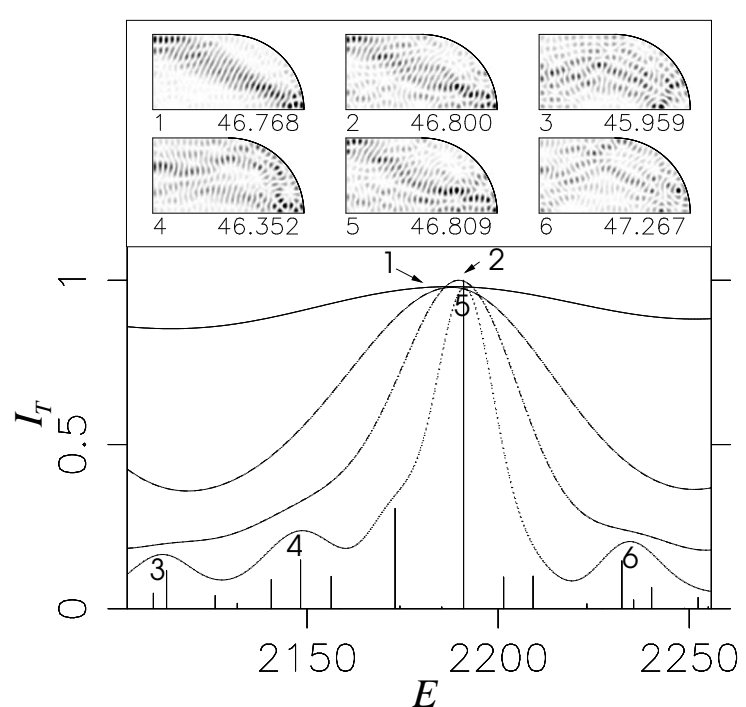

FIG. 3. Enlargement of the region of the spectrum of Fig. 2 corresponding to the $9-$ th band. The smoothed version has been computed for three different values of the resolution time, T: 0.02 (full line), 0.04 (dashed line), 0.07 (dot-dashed line) and 0.135 (dotted line). In the upper part, the wave functions associated to the labeled bands are presented. The corresponding values of $\sqrt{\langle E\rangle}$ are shown below each plot.

As can be seen, the wave function labeled by 2 , corresponding to $T=0.07$, is very similar to that already presented in Fig. 2 that was computed at $T=0.04$. However, function 1, calculated at a smaller time, presents a much stronger localization along the diagonal PO, being better defined specially at the corners. This is due to the fact that a smaller resolution time allows the participation of more eigenstates in the expansion of the wave function associated to each band. Finally, at the largest time considered, $T=0.135$, the band splits into four components, whose associated wave functions are shown in plots 3 to 6 of Fig. 3. By visual inspection, these functions are found to be scarred by POs (d), (c), (b)/(a) and (d), respectively. This assignment was confirmed more quantitatively in the following way. In the first place, we constructed localized wave functions on the scarring orbits in the same way described above for the diagonal PO (see Fig. 2), and calculated the overlaps of the resulting functions with those of the intrabands considered. The wave function associated to peak number 5 is a linear combination $(56 \%$ and $42 \%)$ of the structures localized on orbits (b) and (a), being the corresponding overlap values 0.75 and 0.65 , respectively. Values greater than 0.7 were obtained for the other three peaks. In the second place, the corresponding calculated values of $\sqrt{\langle E\rangle}$ : $45.959,46.352,46.809$, and 47.267, agree remarkably well with the predicted values of $k$ that are obtained from the Bohr-Sommerfeld quantization rule [Eq. (6) with the appropriate values for the parameters $L$ and $\nu]$ : 45.966, $46.367,46.748 / 46.715$, and 47.225 . The reason why the 9-th band in the spectrum of Fig. 2 splits into these com- 
ponents can be understood by considering the Hamiltonian matrix elements among localized states 1 and 3-6 of Fig. 3. These numbers, defined as interactions in Ref. [8], when calculated $(226,664,1706$ and 533 respectively) turn out to be larger than any other interaction with localized structures in this energy region. The picture that emerges is analogous to that of non-overlapping resonances embedded in a continuum (see discussion in Ref. [15]). The band or intraband structures obtained with Eq. (5) correspond, loosely speaking, to states dynamically averaged over classical paths. They are initially located in the neighbourhood of unstable POs (equivalent to a decaying resonance) which are the only renmant of order in the middle of the surrounding chaotic sea (playing the role of the continuum). Moreover, the corresponding spectra present a great resemblance with resonance spectra, and the rhs of Eq. (5) can be viewed as an approximate projection operator (on each resonance) acting on $|\phi(0)\rangle$.

The same analysis can be applied to investigate the intraband structure of other bands in the spectrum of Fig. 2, in order to discover the signature of other POs. This is the case, for example, of the wave functions presented in Fig. 1, which are scarred by POs (e) and (f), respectively. They correspond to peaks in the intraband structure of bands 12 and 13 of Fig. 2. Again the computed values of $\sqrt{\langle E\rangle}: 50.282$ and 52.757 , agree very well with the predicted values of $k: 50.245$ and 52.722 .
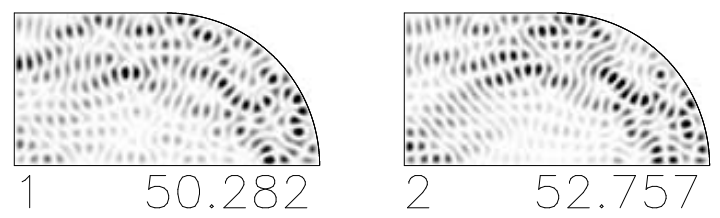

FIG. 4. Wave functions corresponding to different components of bands 12 and 13 in the spectra of Fig. 2 , showing localization along periodic orbits (e) and (f) of Fig. 1. The corresponding values of $\sqrt{\langle E\rangle}$ are shown below each plot.

This result indicates that the intraband structure associated to different bands can be quite distinct. As shown, this is due to the fact that all POs involved in a given intraband pattern (corresponding to a given smoothing time) quantize in different ways, so that each one gives rise to a characteristic spacing. It is the relation among the values of these spacings and that of the original orbit (the LUPO in our case), which determines the distribution of new peaks in each band. Since they are in principle unconnected, the pattern under each band will be different.

In conclusion, we have presented a study of the scarring effect of POs beyond the short time dynamics in a very chaotic system. A few POs, other than the LUPO, have been shown to be able to develop their influence in the low resolution features of the corresponding spectra or local density of states, appearing at the associated quantized energy values; in other words they are responsible for the averaged dynamics in this time range. It has also been described how localized wave functions can be constructed for this intermediate time domain. These results provide a good smoothed picture around a given $\mathrm{PO}$ and the family of orbits connected to it, and then constitute an important first step in order to fully disentangle the complexity involved in the eigenvalues spectrum of very chaotic systems.

This work has been supported in part by DGES (Spain) under Projects No. PB95-425 and PB96-76. DW gratefully acknowledges support from Agencia Española de Cooperación Internacional (Spain) and FOMEC (Argentina) for his stay in Madrid.

[1] M. C. Gutzwiller, Am. J. Phys. (Resource Letter), 66, 304 (1997).

[2] O. Bohigas, M.--J. Giannoni, and C. Schmit, Phys. Rev. Lett. 52,1 (1984).

[3] L. Kaplan, Nonlinearity 12, R1 (1999).

[4] E. J. Heller, Phys. Rev. Lett. 53, 1515 (1984).

[5] S. Tomsovic and E. J. Heller, Phys. Rev. Lett. 67, 664 (1991).

[6] T. Takami, Phys. Rev. Lett. 68, 3371 (1992).

[7] F. J. Arranz, F. Borondo, and R. M. Benito, Phys. Rev. Lett. 80, 944 (1998).

[8] E. Vergini and D. A. Wisniacki, Phys. Rev. E 58, R5225 (1998).

[9] L. Kaplan and E. J. Heller, Phys. Rev. E 59, 6609 (1999).

[10] G. G. de Polavieja, F. Borondo and R. M. Benito, Phys. Rev. Lett. 73, 1613 (1994).

[11] E. J. Heller, in Chaos and Quantum Physics, edited by M. J. Giannoni, A. Voros, and J. Zinn-Justin (Elsevier, Amsterdam, 1991).

[12] L. Kaplan, Phys. Rev. Lett. 80, 2582 (1998); L. Kaplan and E. J. Heller, Ann. Phys. (N.Y.) 264, 171 (1998).

[13] E. Vergini and M. Saraceno, Phys. Rev. E 52, 2204 (1995).

[14] D. Wisniacki, F. Borondo, E. Vergini, and R. M. Benito, in preparation.

[15] J. M. Gomez-Llorente, F. Borondo, N. Berenguer, and R. M. Benito, Chem. Phys. Lett. 192, 430 (1992). 